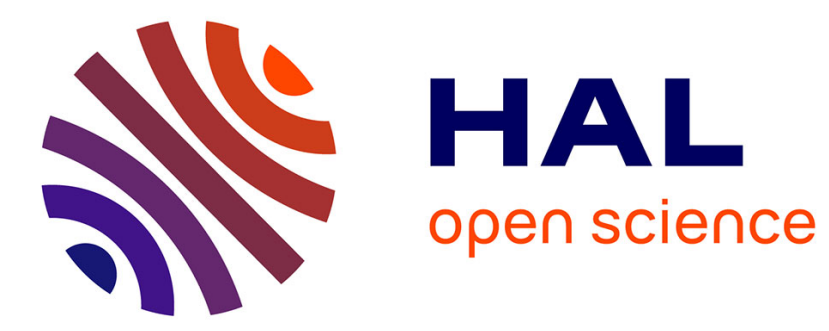

\title{
Beta spectrum measurements using a quasi- $4 \pi$ detection system based on Si detectors
}

\author{
A. Singh, X. Mougeot, B. Sabot, D. Lacour, A. Nourreddine
}

\section{To cite this version:}

A. Singh, X. Mougeot, B. Sabot, D. Lacour, A. Nourreddine. Beta spectrum measurements using a quasi- $4 \pi$ detection system based on Si detectors. Appl.Radiat.Isot., 2019, 154, pp.108897. 10.1016/j.apradiso.2019.108897 . hal-02362184

\section{HAL Id: hal-02362184 \\ https://hal.science/hal-02362184}

Submitted on 19 Nov 2019

HAL is a multi-disciplinary open access archive for the deposit and dissemination of scientific research documents, whether they are published or not. The documents may come from teaching and research institutions in France or abroad, or from public or private research centers.
L'archive ouverte pluridisciplinaire HAL, est destinée au dépôt et à la diffusion de documents scientifiques de niveau recherche, publiés ou non, émanant des établissements d'enseignement et de recherche français ou étrangers, des laboratoires publics ou privés. 


\title{
Beta spectrum measurements using a quasi-4 $\pi$ detection system based on $\mathrm{Si}$
}

\section{detectors}

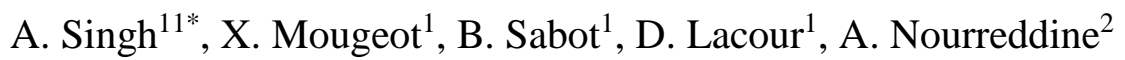

${ }^{1}$ CEA, LIST, Laboratoire National Henri Becquerel (LNE-LNHB), CEA-Saclay 91191, Gif/Yvette Cedex, France

${ }^{2}$ Institut Pluridisciplinaire Hubert Curien, University of Strasbourg, CNRS-IN2P3-UMR 7178, 23 rue du Loess, BP 28, F-67037, Strasbourg Cedex 2, France

\begin{abstract}
The accurate measurement of beta spectra is highly important in numerous fields such as nuclear energy, nuclear medicine, ionizing radiation metrology and fundamental physics. We have developed a beta spectrometer close to $4 \pi$ configuration based on silicon detectors. The influence of self-absorption has been studied by means of Monte Carlo simulations and the source preparation technique has been optimized consequently. The measured spectra from ${ }^{133} \mathrm{Ba}$ and ${ }^{36} \mathrm{Cl}$ decays have been compared with PENELOPE simulations.
\end{abstract}

\section{Introduction}

Since 1970, the shape of the beta spectrum has scarcely been studied but there is a growing demand from several users in the scientific community. The nuclear energy industry appeals to precisely study the shape of beta spectra for the calculation of decay heat, and the fundamental research community for understanding measured antineutrino spectra from nuclear reactors. In nuclear medicine, beta-emitters are used increasingly for imaging (e.g. ${ }^{67} \mathrm{Cu},{ }^{90} \mathrm{Y},{ }^{32} \mathrm{P}$ etc.) and internal

\footnotetext{
${ }^{1}$ Corresponding author.

E-mail address: abhilasha.singh@cea.fr
} 
radiotherapy (Bardies and Chatel, 1994). The ionizing radiation metrology requires the beta spectrum shape for the activity measurement of radionuclides carried out by liquid scintillation counting techniques, using either the TDCR (triple-to-double coincidence ratio) or the CIEMATNIST techniques (Broda et al., 2007). Precise and validated beta shapes are needed to reduce uncertainties.

Few years ago, the Laboratoire National Henri Becquerel (LNHB), the primary laboratory for ionizing radiations in France, developed a theoretical code named "BetaShape" which calculates the beta spectra of allowed and forbidden unique transitions (Mougeot, 2017). This code handles ENSDF (Evaluated Nuclear Structure Data Files) standard files (NNDC, 2019), includes a database of experimental shape factors (Mougeot, 2015) and takes into account the atomic screening effect and radiative corrections. The calculations are more complicated in the case of forbidden non-unique transitions because the nuclear structure contribution cannot be clearly separated from the lepton dynamics. The BetaShape code then treats such a transition according to the usual $\xi$ approximation, i.e. as an allowed or a forbidden unique transition with the same change of total angular momentum.

An experimental study of all beta-decaying radionuclides is in practice impossible, but measuring some of them accurately is essential. This will allow an experimental validation of the theoretical predictions, both for constraining them and for assessing their predictive power. Some measurements have previously been performed using a single $\mathrm{Si}$ detector in a less than $2 \pi$ configuration at LNHB (Bisch et al., 2014). The measured spectrum was found highly distorted because of scattering and backscattering phenomena. In principal, the measured spectrum can be unfolded with several different methods using the response function of the detection system. However, this type of correction brings about a high uncertainty from the unfolding process. In 
order to reach better uncertainties, it is necessary to limit the sources of distortion arising from the detection system. Therefore, we have developed a beta spectrometer based on Si detectors in an almost $4 \pi$ configuration. The measured spectrum has been compared with very precise Monte Carlo simulations performed with the PENELOPE code (Salvat, 2015). Special care has been taken to reduce the distortion due to the self-absorption of beta particles within the source, and the radioactive source preparation for the beta spectrometry with VYNS film is mentioned in section 3. The necessity of the removal of the remaining distortion is discussed.

\section{Experimental set-up}

The experimental configuration with a source sandwiched between two planar silicon detectors is illustrated in Fig. 1. The current detection system has been designed to minimize all the sources of spectral distortion, which include the detector configuration, the thickness of the radioactive source, the detector dead layer etc. The basic concept of this close $4 \pi$ geometry is to get the sum of the energy deposited in each detector for every emitted particle, a conventional coincidence procedure. This detection system is comprised of two identical PIPS ${ }^{\circledR}$ (Passivated Implanted Planar Silicon) detectors from Mirion Technologies, each $1 \mathrm{~mm}$ thick (electrons of maximum energy up to $850 \mathrm{keV}$ can have full energy deposition) with an active surface area of $300 \mathrm{~mm}^{2}$. According to the information furnished by the manufacturer, the detectors have a dead layer of $50 \mathrm{~nm}$ Si-equivalent. In principle, the Si detectors are operational at room temperature, although the thermal leakage current can add noise and reduce the signal-to-noise ratio. Therefore, we have designed the device to be operational at liquid nitrogen temperature. To operate the device at such a low temperature, it is maintained under ultra-high vacuum, which also prevents scattering in air. The device consists of two detector holders made in copper, which encompass the radioactive 
source in the middle. The radioactive source is deposited on top of a VYNS copolymer film and is facing the top Si detector. The distance between the two Si surfaces is $3 \mathrm{~mm}$ and this is why it is called a quasi-4 $4 \pi$ detection system. As each detector is encapsulated by the manufacturer in a metal housing (with a radial microdot connector), it is very difficult to further reduce the distance between the detectors. The detector holders have a copper plate to accommodate preamplifiers for each detector, as shown in Fig. 2.

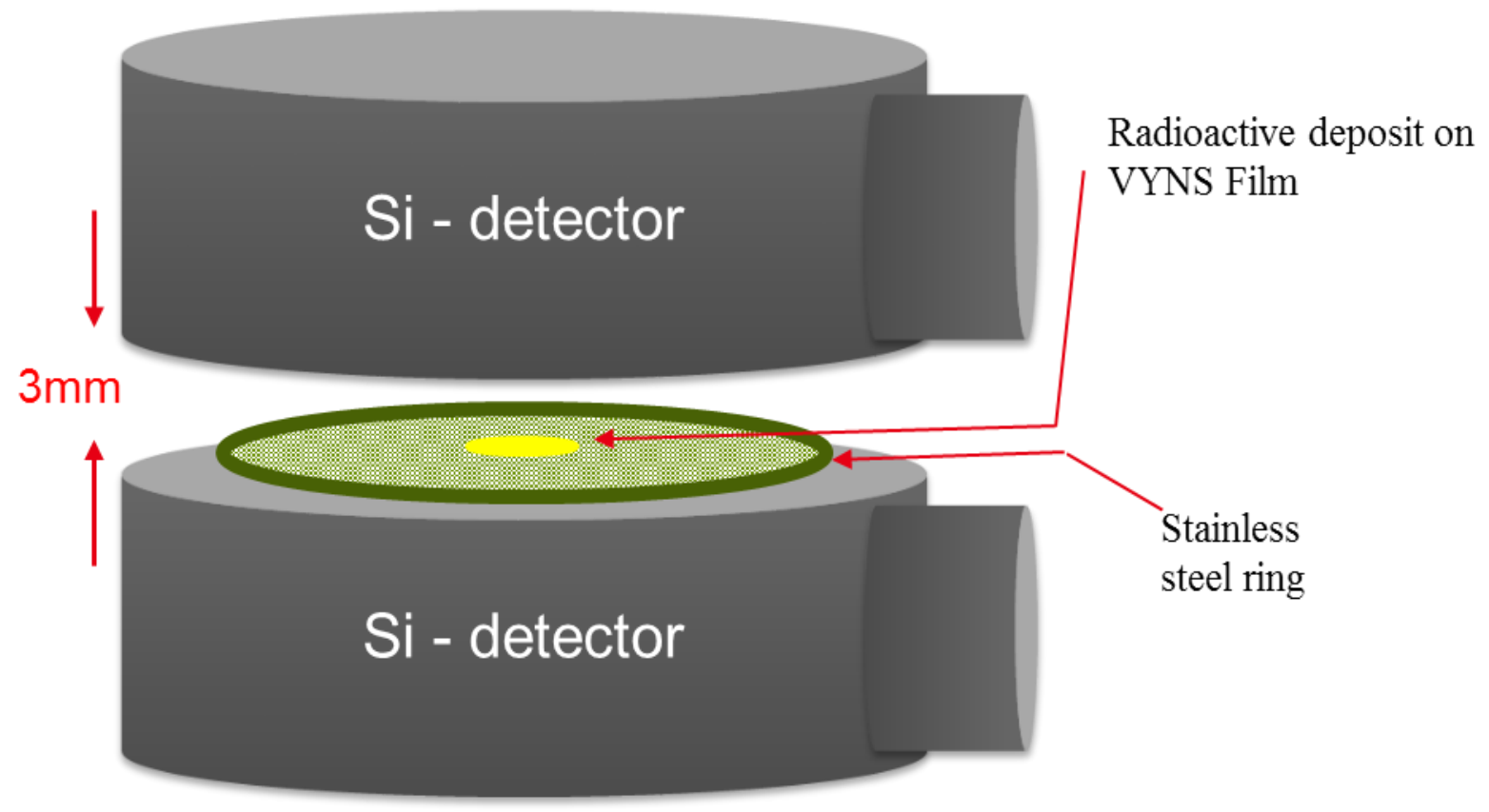

Figure 1. Basic scheme of the device configured close to a $4 \pi$ geometry with two PIPS ${ }^{\circledR}$ detectors.

A charge-sensitive preamplifier $1422 \mathrm{H}$ from $\mathrm{CAEN}$ is used for each detector. This device is fixed inside the detection chamber on a cold copper finger in thermal contact with the liquid nitrogen dewar in the detection chamber, which is an aluminum cube with an edge size of $17 \mathrm{~cm}$ (Bisch et al., 2014). In order to maintain the vacuum, the high voltage and power are supplied through a customized electronic feedthrough. The position of the preamplifiers on the copper plates 
minimizes the distance between each detector and its preamplifier in order to reduce the capacitance and thus to reach better energy resolution and detection threshold. In addition, the operation of the preamplifiers at a lower temperature decreases the thermal noise. However, the maintenance of vacuum by the turbo pump adds additional noise through vibrations, which were suppressed by placing the pump tube in a sand box.

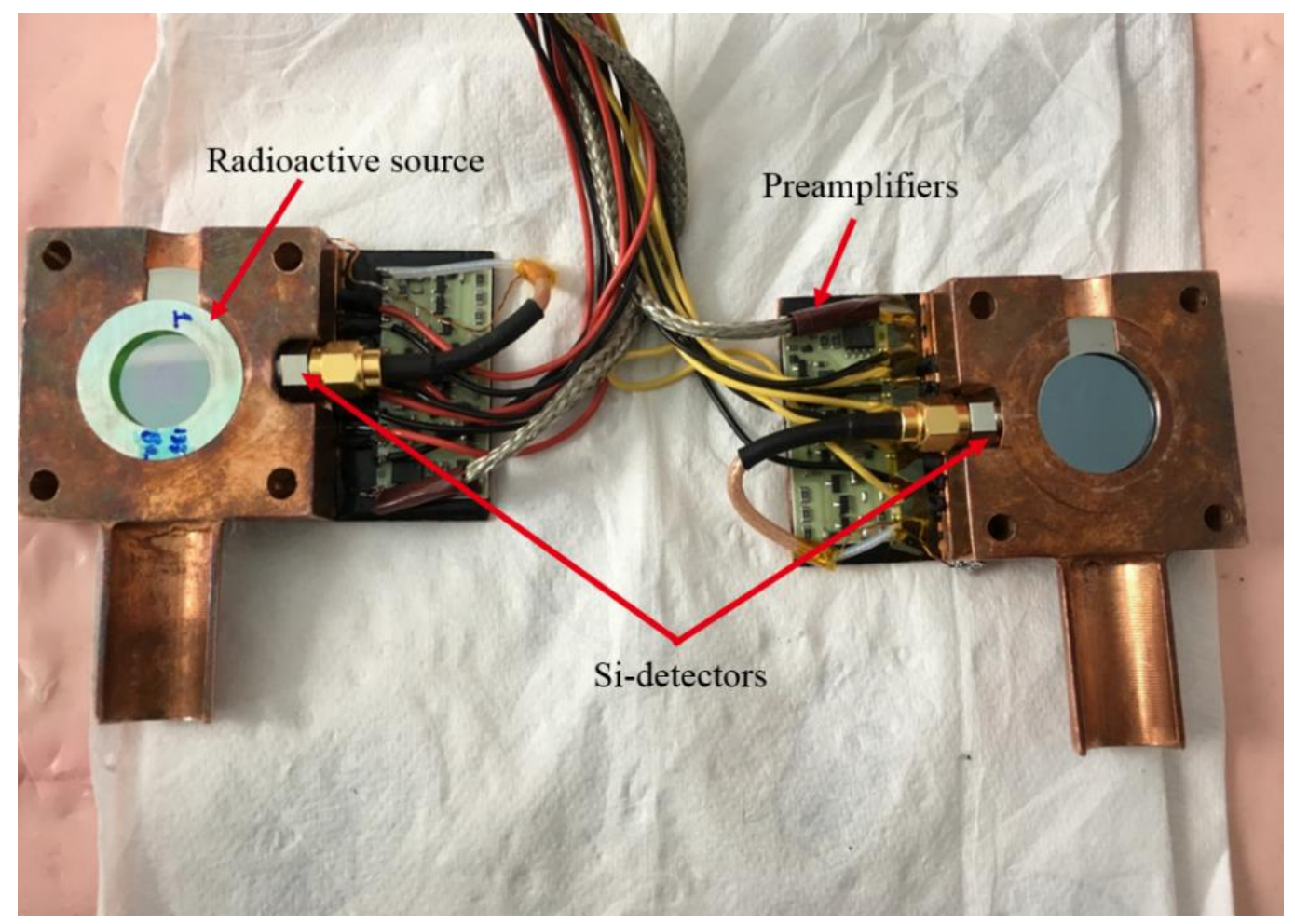

Figure 2. Experimental set-up with two PIPS ${ }^{\circledR}$ detectors $\left(300 \mathrm{~mm}^{2}\right)$ in a configuration close to $4 \pi$ geometrv.

\section{Radioactive source preparation}

Knowledge of the spectral distortion due to the self-absorption of the beta particles within the radioactive source is critical for beta spectrometry. The effect of the source thickness on selfabsorption is well known from the 1950s (Fulbright and Milton, 1951; Wu and Feldman, 1949 and 1951). Consequently, it is necessary to use thin sources for beta spectra studies to reduce the selfabsorption and diffusion phenomena. 
The radioactive sources have been prepared following four main steps:

1. Preparation of the VYNS films as source carrier;

2. Electrospraying of latex microspheres as seeding agents;

3. Deposition of the radioactive solution on the pre-treated supports;

4. Drying of the source drops.

The VYNS film is supported on a stainless steel ring (outer diameter is $25 \mathrm{~mm}$; inner diameter is $15 \mathrm{~mm}$; and thickness is $0.15 \mathrm{~mm}$ ). The electrospraying of latex microspheres has been used to obtain a homogeneous radioactive deposit (10 $\mathrm{mm}$ diameter and $0.15 \mu \mathrm{m}$ thickness) with many small crystals. The activity of the radioactive deposit is typically $1 \mathrm{kBq}$ which limits the pulse pileup as the decay constant of signal is typically $50 \mu$ s. Additionally, a shaping parameterization of trapezoidal signal inherently accounts for pile-up rejection in our digital acquisition system.

Monte Carlo studies with the PENELOPE code, described in the next section, have been performed to quantify the distortion of the spectral shape due to energy loss, especially at the lowest beta particle energies. As expected, the shape of the spectrum was found to be closer to the theoretical prediction, used as an input of the simulation, with a $0.5 \mu \mathrm{m}$ thin VYNS film source and more distorted with an $18 \mu \mathrm{m}$ Mylar ${ }^{\circledR}$ film source.

\section{Monte Carlo simulations}

\subsection{Description}

The detailed experimental configuration of the detection system has been implemented in the PENELOPE code, the 2014 version (Salvat, 2015). It is a well-known Monte Carlo simulation code of coupled electron-photon interactions that simulates the transport of electrons and photons 
in homogeneous materials of different chemical compositions within an energy range of $50 \mathrm{eV}$ to $1 \mathrm{GeV}$ (Salvat, 2012).

As electrons are light particles and may lose energy in the interactions within the detection system, a detailed and precise description of the system is necessary. As we have designed the device by ourselves, we have an accurate knowledge of the dimensions of each component involved. The dimensions for the Si detectors are used as provided by the manufacturer, which are approximate. The PIPS ${ }^{\circledR}$ are $1 \mathrm{~mm}$ thick, have a $50 \mathrm{~nm}$ thick dead layer and an active surface area of $300 \mathrm{~mm}^{2}$. The radioactive source is modeled as a $0.5 \mu \mathrm{m}$ thick VYNS film supported by a stainless steel ring as described in the previous Section. The radioactive deposit in the simulation is modeled as a volume of air at pressure $10^{-7} \mathrm{mbar}(10 \mathrm{~mm}$ diameter and $10 \mathrm{~nm}$ thickness $)$ on the top of a plain latex layer of $8 \mathrm{~nm}$ thickness. This layer of latex models the electrosprayed latex microspheres, whose actual diameter is $70 \mathrm{~nm}$. Knowing the area of the deposit, an estimate of the weight of the latex layer is possible. By weighing the films before and after electrospraying, we deduced that we effectively deposited a monolayer of microspheres. The actual volume of latex is the deposit area paved with spheres. Keeping the same volume, we deduced the effective thickness of $8 \mathrm{~nm}$ for an equivalent plain layer. The homogeneity of the source is assumed by the SOURCE code in PENELOPE, and has been confirmed by autoradiography.

In this work, we have studied the shape of the measured beta spectra by simulating the experimental geometry for a few beta-emitting radionuclides. For this, we have used the beta spectra computed with the BetaShape code (Mougeot, 2017) as source input.

In addition, we have modified the PENELOPE code to obtain the deposited energy in each detector event by event. This allows us to get the sum of the deposited energy in both detectors for each event and to perform some post-simulation analysis. 


\subsection{Results}

A precise description of the experimental geometry presented above is highly critical. First, we performed PENELOPE simulations to study the effect of the most important parameter in our configuration, i.e. the distance between the two $\mathrm{Si}$ surfaces. The ${ }^{14} \mathrm{C}$ beta spectrum from BetaShape was used as a source input of the simulation. As shown in Fig. 3, the distance between the active layers of the detectors has a significant influence on the shape of the aggregate beta spectrum. The current minimum distance is $3 \mathrm{~mm}$ due to mechanical constraints. However, the simulation clearly shows that it is needed to decrease even more this distance in order to obtain a measured spectrum as close as possible to the emitted spectrum.

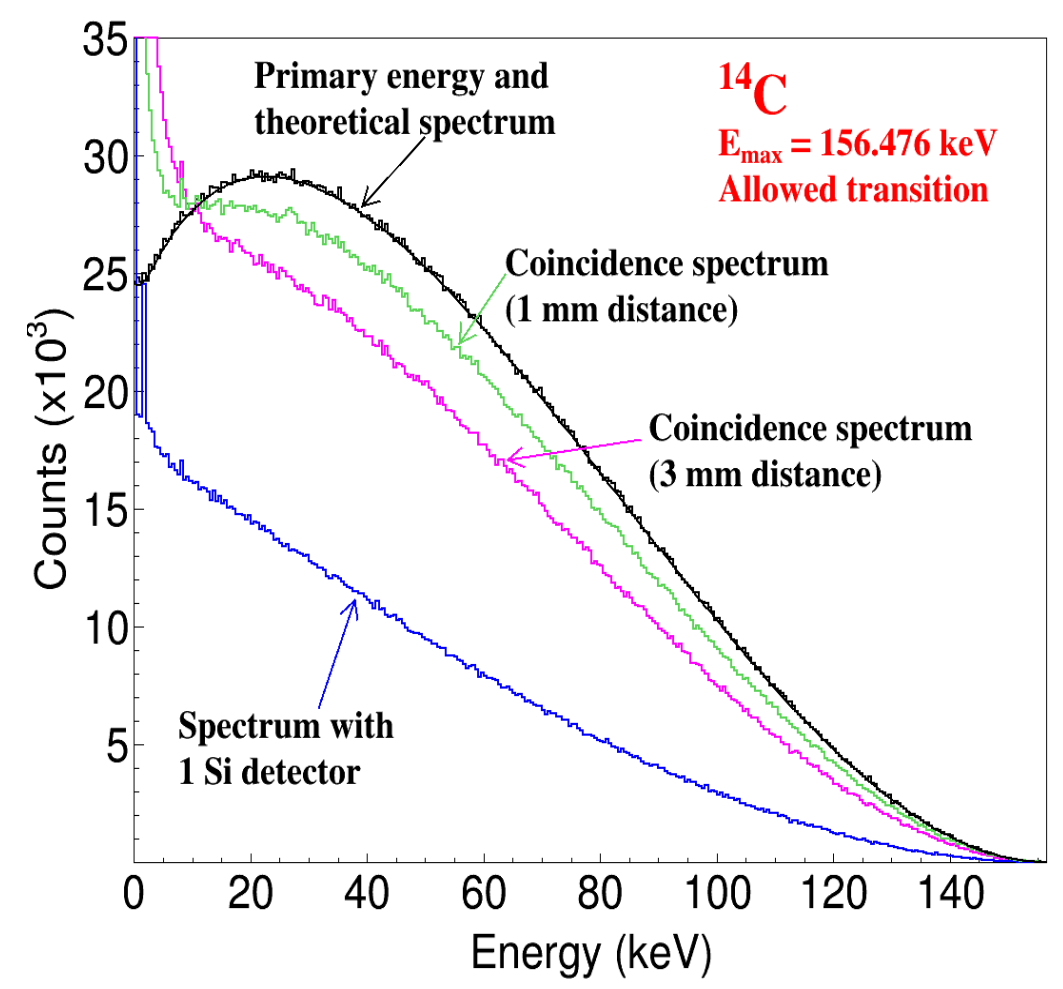

Figure 3. The Monte Carlo study showing the influence of the distance between the detectors. The current set-up configuration is $3 \mathrm{~mm}$. 
Furthermore, simulations were performed for the optimization of the experimental setup in terms of the source substrate. We have simulated two realistic thin films as a source support for the radioactive deposit, a $0.5 \mu \mathrm{m}$ thick VYNS film and an $18 \mu \mathrm{m}$ thick Mylar ${ }^{\circledR}$ film. As shown in Fig. 4, the self-absorption in the VYNS film is less than in the Mylar film and this effect is more significant at low energies. As a result, we have used $0.5 \mu \mathrm{m}$ thick VYNS films as the source support material.

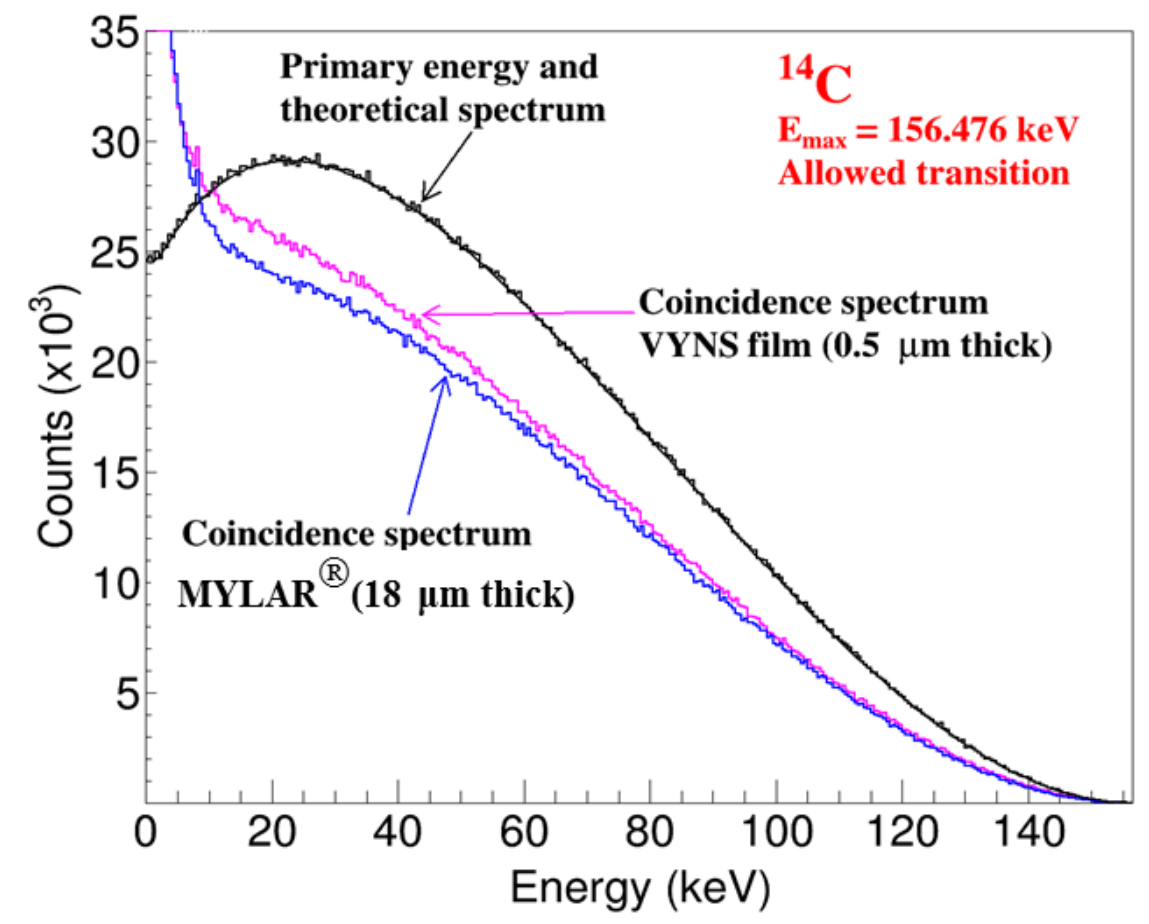

Figure 4. Monte Carlo study of the self-absorption phenomenon in the current measurement system.

\section{Preliminary measurements}

\section{$5.1^{133} \mathrm{Ba}$}

The energy calibration of the detectors was carried out with the measurement of a ${ }^{133}$ Ba source. The response of the detection system was found to be linear from $40 \mathrm{keV}$ to $350 \mathrm{keV}$. The curve in blue shown in Fig. 5 is the measured spectrum of conversion electrons of ${ }^{133} \mathrm{Ba}$. The red curve 
is the simulated spectrum from the PENNUC module of the PENELOPE code, which uses evaluated decay data from DDEP (García-Toraño, 2017). PENELOPE does not take into account the charge collection in the detector and the electronic noise, which both affect the energy resolution of the system. Therefore, the simulated spectrum has been convoluted with a Gaussian distribution using the experimentally observed standard deviation. The FWHM (Full Width at Half Maximum) was observed to be $16 \mathrm{keV}$ for the electron peak of ${ }^{133} \mathrm{Ba}$ at $80 \mathrm{keV}$. The energy threshold was $35 \mathrm{keV}$. The measured and simulated spectra are in fair agreement. It is evident that both the energy resolution and the energy threshold can be drastically improved and we are working on further improvements, especially related to the electronics of our detection system.

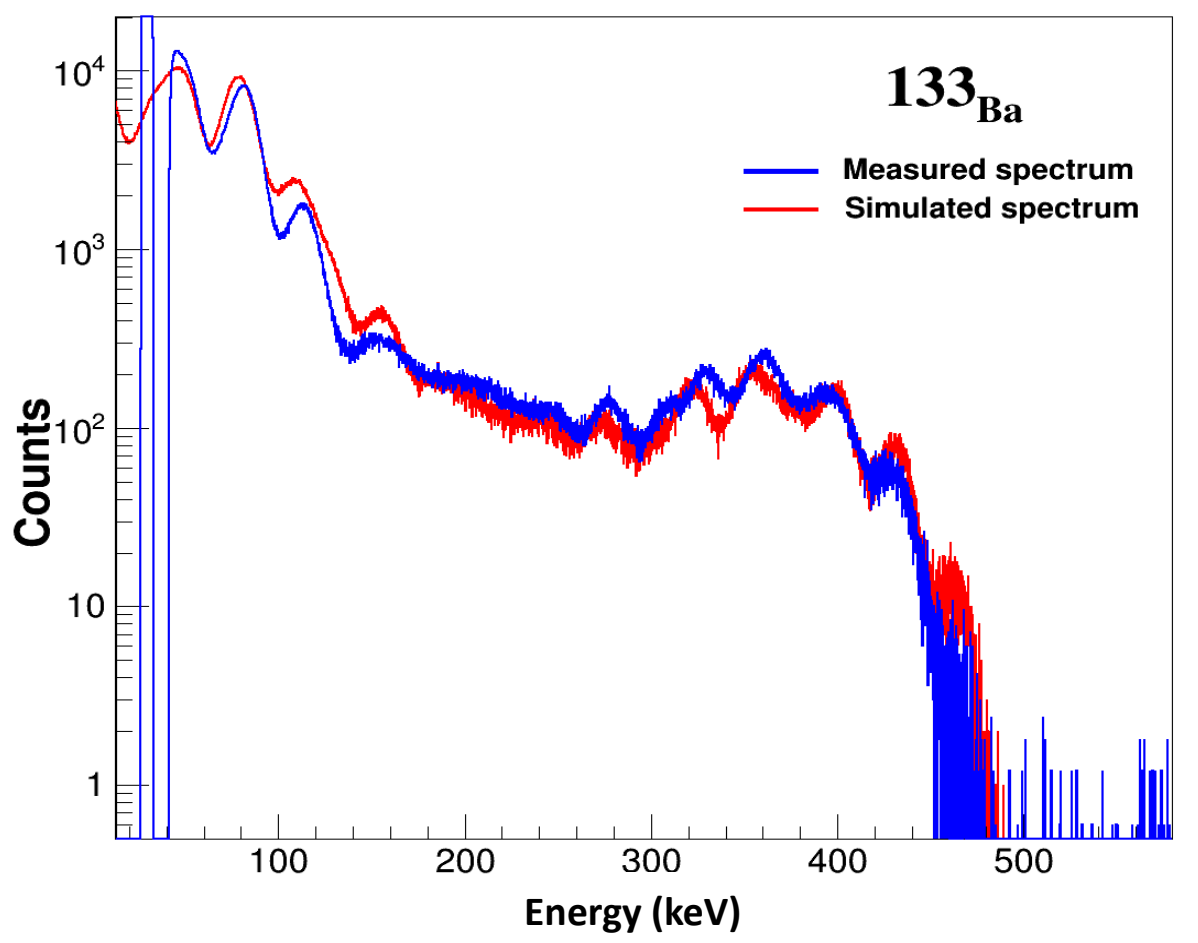

Figure 5. Comparison of measured spectrum and simulated spectrum using PENNUC module of conversion electrons of ${ }^{133} \mathrm{Ba}$. 


\section{$5.2{ }^{36} \mathrm{Cl}$}

The beta spectrum from ${ }^{36} \mathrm{Cl}$ decay was also measured. With a maximum energy of 709.53(4) keV (Wang et al., 2017), an emitted beta particle should be stopped within the thickness of Si detectors. The ${ }^{36} \mathrm{Cl}$ source was prepared on VYNS film and the corresponding experimental geometry has been simulated in PENELOPE. This transition is second forbidden non-unique and thus sensitive to the structure of the nuclear states involved, making an accurate theoretical prediction difficult. Therefore, the input spectrum was determined using the experimentally extracted shape factor from Rotzinger (Rotzinger et al., 2008). The energy calibration curve determined from the ${ }^{133} \mathrm{Ba}$ measurement was applied to the ${ }^{36} \mathrm{Cl}$ measurement, assuming a linear response beyond $400 \mathrm{keV}$, which is reasonable for Si detectors. The spectra were normalized by integration between $200 \mathrm{keV}$ and $300 \mathrm{keV}$ and are given in Fig. 6. Above $35 \mathrm{keV}$, an excellent agreement was observed between the measured and simulated spectra, which demonstrates the relevance of the present approach with Si detectors.

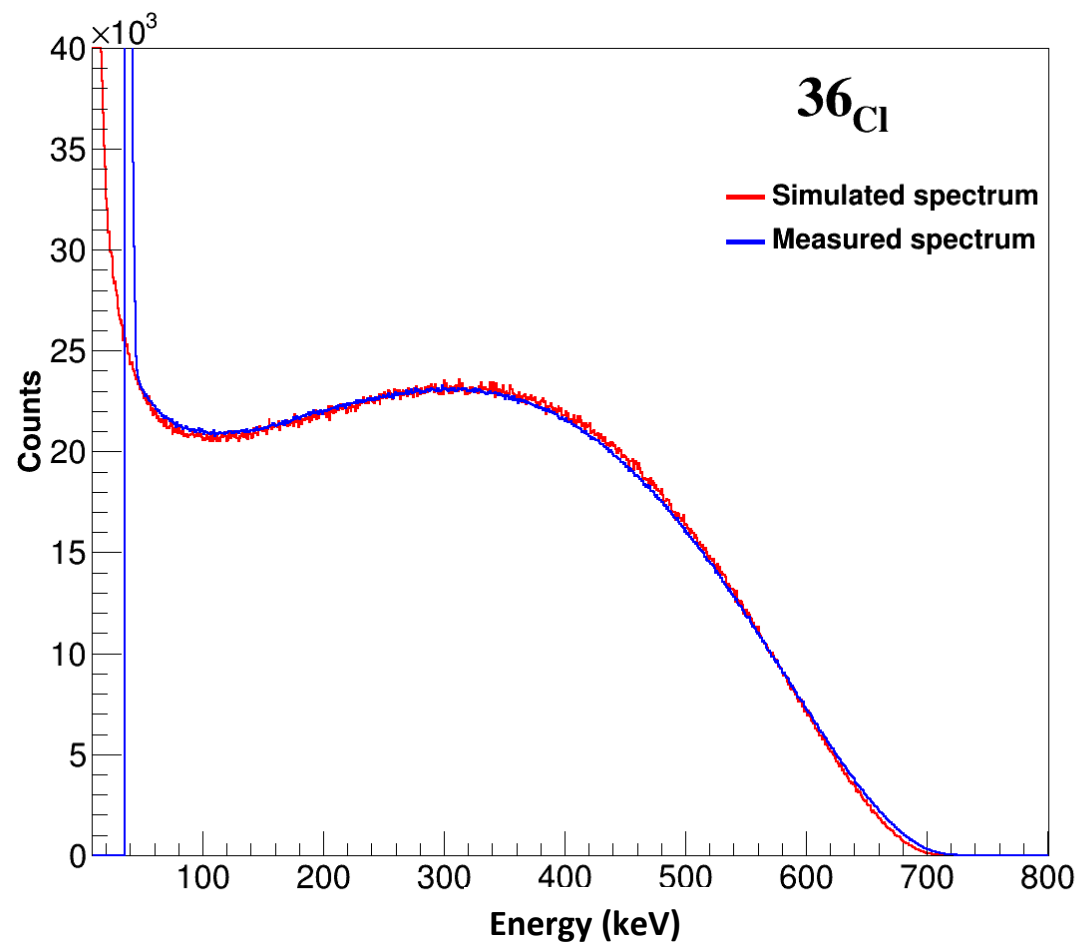

Figure 6. Comparison of measured spectrum and simulated spectrum for ${ }^{36} \mathrm{Cl}$ decay ( $2{ }^{\text {nd }}$ forbidden non-unique transition). 


\section{Conclusion}

Our measurement device, configured close to $4 \pi$ geometry, dedicated to the measurement of beta spectra is operational and some preliminary measurements have been successfully carried out. The influence of the source on the spectrum shape has been studied and the need for thin radioactive sources was justified. Monte Carlo simulations using the PENELOPE code were in good agreement with the preliminary measurements. However, there are still some issues with the noise coming from the associated electronics, and maybe from the detectors themselves. Nevertheless, the preliminary results of the presented work promise to deliver useful measurements of beta spectra with this technique.

As demonstrated, the current incomplete $4 \pi$ geometry does not avoid significant spectral distortions. They can be compensated for through an unfolding process currently in development. It will allow extracting the experimental shape factors and assessing firm uncertainties.

\section{References}

Bardies, M., Chatal, J.-F., 1994. Absorbed doses for internal radiotherapy from 22 beta-emitting radionuclides: beta dosimetry of small spheres. Phys. Med. Biol. 39, 961.

Broda, R., Cassette, P., Kossert, K., 2007. Radionuclide metrology using liquid scintillation counting. Metrologia 44, S36.

Bisch, C., Mougeot, X., Bé, M.-M., Nourreddine, A.-M., 2014. Development of a system for measuring the shape of spectra using a semiconductor Si detector. Nuclear Data Sheets 120C, 95.

Fulbright, H. W., Milton, J. C. D., 1951. The beta-spectrum of ${ }^{36} \mathrm{Cl}$. Phys. Rev. 82, 274. 
García-Toraño, E., Peyres, V., Bé, M.-M., Dulieu, C., Lépy, M.-C., Salvat, F., 2017. Simulation of decay processes and radiation transport times in radioactivity measurements. Nucl. Instr. and Meth. in Phys. Res. B 396, 43-49.

Mougeot, X., 2015. Reliability of usual assumptions in the calculation of $\beta$ and $v$ spectra. Phys. Rev. C 91, 055504.

Mougeot, X., 2017. BetaShape: A new code for improved analytical calculations of beta spectra. EPJ Web of Conferences 146, 12015.

NNDC, 2019. Bhat, M.R. in Evaluated Nuclear Structure Data File (ENSDF), edited by S. M. Qaim, Nucl. Data Sci. Tech. (Springer-Verlag, Berlin, 1992), p. 817. Data extracted using the NNDC On-Line Data Service from the ENSDF database, files revised as of February 2019. See https://www.nndc.bnl.gov/ensdf/

Rotzinger, H., Linck, M., Burck, A., Rodrigues, M., Loidl, M., Leblanc, E., Fleischmann, L., Fleischmann, A., Enss, C., 2008. Beta Spectrometry with Magnetic Calorimeters. J. Low Temp. Phys. 151, 1087-1093.

Salvat, F., et al., 2009. PENELOPE-2008: A Code System for Monte Carlo Simulation of Electron and Photon Transport. NEA-OECD (2009).

Salvat, F. 2015. PENELOPE-2014 - A Code System for Monte Carlo Simulation of Electron and Photon Transport. NEA report NEA/NSC/DOC (2015).

Wang, M., Audi, G., Kondev, F. G., Huang, W. J., Naimi, S., Xu, X., 2017. The AME2016 atomic mass evaluation. Chin. Phys. C 41, 03003.

Wu, C. S., Feldman, L., 1949. The Beta-Ray Spectrum of Chlorine ${ }^{36}$. Phys. Rev. 76, 693-695.

Wu, C. S., Feldman, L., 1951. Beta-Spectrum of $\mathrm{Cl}^{36}$. Phys. Rev. 82, 457-458. 